Research Article

\title{
Adaptive Fault Estimation and Fault Tolerant Control for Polynomial Systems: Application to Electronic and Mechanical Systems
}

\author{
A. Messaoudi, ${ }^{1}$ H. Gassara ${ }^{(D},{ }^{1}$ and A. El Hajjajii ${ }^{2}$ \\ ${ }^{1}$ Laboratory of Sciences and Technology of Automatic Control and Computer Engineering, National School of Engineering of Sfax, \\ University of Sfax, P. O. Box 1173, 3038 Sfax, Tunisia \\ ${ }^{2}$ University of Picardie Jules Verne, MIS Laboratory, UFR Sciences, 33, Rue St Leu, F-80000 Amiens, France
}

Correspondence should be addressed to H. Gassara; gassara.hamdi@yahoo.fr

Received 18 May 2021; Revised 17 June 2021; Accepted 25 June 2021; Published 6 July 2021

Academic Editor: Abdellatif Ben Makhlouf

Copyright (C) 2021 A. Messaoudi et al. This is an open access article distributed under the Creative Commons Attribution License, which permits unrestricted use, distribution, and reproduction in any medium, provided the original work is properly cited.

This paper presents a sum of squares (SOS) approach for active fault tolerant control (AFTC) of nonlinear polynomial systems. A polynomial adaptive fault estimation algorithm for polynomial systems is firstly proposed. Then, sufficient conditions for the existence of the fault estimator are given in terms of SOS which can be numerically (partially symbolically) solved via the recently developed SOSTOOLS. Based on the obtained online fault estimation information, a fault-tolerant control strategy is designed for both compensating the effect of actuator faults in real time and stabilizing the closed-loop system. Finally, tunnel diode circuit and mass-spring-damper systems are used to demonstrate the applicability of the proposed approach.

\section{Introduction}

The growing complexity of modern industrial processes gives rise to increasing demands regarding fault estimation (FE) and fault-tolerant control (FTC). Various FE strategies have been proposed in the literature. For example, proportional integral sliding mode observer has been considered in Elleuch et al. [1]; learning algorithm has been proposed in Jia et al. [2]. In Zhang et al.'s study [3], a fast adaptive actuator fault estimation technique is proposed for linear models. In Tayari et al.'s study [4], two adaptive sliding mode observers are adopted for a class of uncertain linear parameter varying systems.

Since the most practical systems are nonlinear, the adaptive observer approach has been extended to deal with nonlinear models. The well-known Takagi Sugeno (TS) fuzzy model has attracted much attention in the past two decades due to its powerful capability to approximate complex nonlinear systems. In Ichalal et al.'s study [5], the state and the actuator faults are well estimated by using the fast adaptive observer proposed in Zhang et al. [3] for TS fuzzy systems. An adaptive observer-based FTC has been designed for a class of TS fuzzy systems with both actuator and sensor faults in Kharrat et al. [6]. Moreover, the $H_{\infty}$ performance is used in this work to attenuate the external disturbance effect.

Polynomial system is a class of nonlinear systems in the form of $\dot{x}=A(x) x+B(x) u$, where $A(x)$ and $B(x)$ are polynomial matrices. This class of systems can describe many engineering systems such as electronic circuits, mechanical systems, and communications systems. For instance, the electronic circuit with tunnel and the massspring-damper are described by polynomial models in Zhao et al. [7] and Li et al. [8], respectively. In Zhao et al.'s study [7], a functional observer is designed in order to estimate the system state and the unknown input, in which the observer design scheme has been applied to electronic circuit with tunnel described by the polynomial model. When this original model is considered, the LMI-based analysis approach cannot be applied directly. For this reason, the control of this electronic circuit is based on the transformation of the original polynomial model into TS fuzzy form. In this case, the controller is designed using LMI-based 
approach. Similarly, a mass-spring-damper system is used by $\mathrm{Li}$ et al. [8] to show the applicability of the proposed controller design.

The latest developments in sum of squares (SOS) programming techniques make it possible to deal directly with polynomial systems. So far, extensive results have been presented for investigating different classes of polynomialbased systems such as polynomial systems, positive polynomial systems, polynomial fuzzy systems, and polynomial fuzzy systems with time delay. Topics on delay-free case cover a wide range including stability analysis by Han et al. [9], stabilization by Zhao et al. [10], fuzzy observer design by Liu et al. [11], passive fault tolerant control by Ye et al. [12], and fault detection filter design by Chibani et al. [13]. Recently, in the direction of investigating several classes of time delay polynomial systems, some results have been proposed in the literature, e.g., stabilization by Gassara et al. [14]; control under actuator saturation by Gassara et al. [15], and observer-based control for positive polynomial systems with time delay by Iben Ammar et al. [16]. These various results are presented in terms of sum of squares (SOS), in which conditions are numerically (partially symbolically) solved via the recently developed SOSTOOLS by Prajna et al. [17]. These results clearly demonstrate that the SOS approach can be used as an effective alternative technique to the LMIbased approaches for nonlinear systems with polynomial matrices. However, to our knowledge, there are no results for adaptive observer-based FTC for polynomial-based systems. Motivated by the aforementioned observation, in this work, the adaptive fault tolerant control problem for a class of polynomial model with actuator faults is investigated, in which polynomial terms depend only on the measurable variables.

The main contributions of this paper can be summarized as follows:

(i) A novel polynomial adaptive observer is proposed. Despite, standard adaptive observer has been extensively studied in literature for fault estimation, polynomial adaptive observer is not yet investigated for the class of polynomial model. The main advantage of the proposed polynomial adaptive observer-based fault estimation compared with the standard one is that the observer gain $L(y(t))$ is not constant but polynomial.

(ii) Various practical engineering systems can be modeled by the proposed polynomial model. For design purpose, the dynamics of these systems are generally approximated in literature by TS fuzzy models. In this case, the polynomial model can reduce the computational load especially when the number of fuzzy rules is high.

(iii) The proposed polynomial model can also increase the modelling accuracy. In fact, we can deal with the original model without using the sector nonlinearity concept to transform the original model into the TS fuzzy model. This allows to avoid setting the variation bounds of some system states.

It becomes increasingly apparent that the SOS approach can be extended to deal with large research topics, e.g., adaptive tracking control by Chen et al. [18] and Wang et al. [19]; event-triggered control by Xie et al. [20]; and finitetime adaptive fault-tolerant control by Wang et al. [21].

This paper is organized as follows. In Section 2, we present a description of a class of polynomial models with actuator faults. Sufficient conditions for the existence of the actuator fault estimator are given in Section 3. These conditions are given in terms of SOS. Meanwhile, based on the online fault estimation, the controller law is then designed to compensate the effect of actuator faults. In Section 4, a tunnel diode circuit and mass-spring-damper systems are presented to demonstrate the applicability of the proposed result. Finally, Section 5 concludes the paper.

\section{Problem Formulation}

Consider the following polynomial model with additive actuator faults:

$$
\left\{\begin{array}{l}
\dot{x}(t)=A(\zeta(t)) x+B(\zeta(t))(u(t)+f(t)) \\
y(t)=C x(t)
\end{array}\right.
$$

where $x(t) \in \mathbb{R}^{n_{x}}$ is the state vector, $u(t) \in \mathbb{R}^{n_{u}}$ is the input vector, $f(t) \in \mathbb{R}^{n_{f}}$ is the additive actuator fault vector, $\zeta(t)$ is available, such as the partial system state variables and the system outputs as in Pang and Zhang's study [22], $y(t) \in \mathbb{R}^{n_{y}}$ is the measurement output vector, $C$ is constant real matrix, and $A(\zeta(t))$ and $B(\zeta(t))$ are polynomial matrices in $\zeta(t)$.

The derivative of $f(t)$ with respect to time is normbounded:

$$
\|\dot{f}(t)\| \leq f_{1 \max } ; \quad 0 \leq f_{1 \max }<\infty .
$$

To estimate actuator faults, the following polynomial adaptive fault diagnosis observer is considered:

$$
\left\{\dot{\hat{x}}(t)=A(\zeta(t)) \hat{x}(t)+B(\zeta(t))(u(t)+\widehat{f}(t))+L(\zeta(t)) e_{y}(t), e_{y}(t)=y(t)-\hat{y}(t), \hat{y}(t)=C \widehat{x}(t)\right.
$$

where $\hat{x}(t) \in \mathbb{R}^{n_{x}}$ is the observer state vector, $\hat{y}(t) \in \mathbb{R}^{n_{y}}$ is the observer output vector, and $\widehat{f}(t) \in \mathbb{R}^{n_{f}}$ is an estimate of actuator fault $f(t)$.

Denote the state and fault estimation errors as follows:

$$
\begin{aligned}
& e_{x}(t)=x(t)-\hat{x}(t), \\
& e_{f}(t)=f(t)-\hat{f}(t) .
\end{aligned}
$$

State estimation error $e_{x}(t)$ is written as 


$$
\dot{e}_{x}(t)=(A(\zeta(t))-L(\zeta(t)) C) e_{x}(t)+B(\zeta(t)) e_{f}(t) .
$$

The conventional adaptive fault estimation algorithm is given by

$$
\dot{\hat{f}}(t)=\Gamma F(\zeta(t))\left(\dot{e}_{y}(t)+\sigma e_{y}(t)\right),
$$

where $\Gamma \in \mathbb{R}^{n_{f} \times n_{f}}$ is the learning rate.

In Figure 1, the block diagram illustrates the proposed polynomial FTC strategy.

From now, to lighten the notation, we will drop the notation with respect to time $t$. For instance, we will employ $x, \hat{x}$, and $\zeta$ instead of $x(t), \hat{x}(t)$, and $\zeta(t)$, respectively.

\section{Main Results}

3.1. Fault Estimation Based on Polynomial Adaptive Algorithm. In this section, the stability of the error dynamics is guaranteed by the following theorem.

Theorem 1. If there exists positive definite matrix $P_{1}$ and polynomial matrices $W_{L}(\zeta)$ and $F(\zeta)$ such that the following SOS optimization problem is feasible.

Minimize $\eta$ subject to

$$
\begin{gathered}
v_{1}^{T}\left(P_{1}-\varepsilon_{1} I\right) v_{1} \text { is SOS, } \\
v_{2}^{T}\left(\Lambda(\zeta)-\varepsilon_{2}(\zeta) I\right) v_{2} \text { is SOS, } \\
-v_{3}^{T}\left(\Xi(\zeta)+\varepsilon_{3}(\zeta) I\right) v_{3} \text { is SOS, }
\end{gathered}
$$

where $v_{1}, v_{2}$, and $v_{3}$ denote vectors that are independent of $x$, $\hat{x}$, and $\zeta$.

$$
\begin{aligned}
& \Lambda(\zeta)=\left[\begin{array}{cc}
\eta I & B^{T}(\zeta) P_{1}-F(\zeta) C \\
* & I
\end{array}\right], \\
& \Xi(\zeta)=\left[\begin{array}{cc}
\xi_{11}(\zeta) & \xi_{12}(\zeta) \\
* & \xi_{22}(\zeta)
\end{array}\right],
\end{aligned}
$$

in which

$$
\begin{aligned}
& \xi_{11}(\zeta)=P_{1} A(\zeta)-W_{L}(\zeta) C+A^{T}(\zeta) P_{1}-C^{T} W_{L}^{T}(\zeta), \\
& \xi_{12}(\zeta)=-\frac{1}{\sigma} A^{T}(\zeta) P_{1} B(\zeta)+\frac{1}{\sigma} C^{T} W_{L}(\zeta) B(\zeta) \\
& \xi_{22}(\zeta)=-2 \frac{1}{\sigma} B^{T}(\zeta) P_{1} B(\zeta)+\frac{1}{\sigma} M
\end{aligned}
$$

then the state estimation error $e_{x}$ and the fault estimation error $e_{f}$ are bounded. Furthermore, if the bound of the first time derivative of $f$ is zero, these variables converge asymptotically to zero. In this case, the gain of the polynomial adaptive observer-based is given by $L(\zeta)=P_{1}^{-1} W_{L}(\zeta)$.

Proof. Consider the following Lyapunov function

$$
V=e_{x}^{T} P_{1} e_{x}+\frac{1}{\sigma} e_{f}^{T} \Gamma^{-1} e_{f} .
$$

Differentiating $V$ with respect to time $t$ and considering (3), (5), and (6), it leads to

$$
\begin{aligned}
\dot{V}= & 2 e_{x}^{T} P_{1}\left((A(\zeta)-L(\zeta) C) e_{x}+B(\zeta) e_{f}\right) \\
& +2 \frac{1}{\sigma} e_{f}^{T} \Gamma^{-1} \dot{f}-2 e_{f}^{T} F(\zeta) C e_{x}-2 \frac{1}{\sigma} e_{f}^{T} F(\zeta) C \dot{e}_{x} .
\end{aligned}
$$

One has

$$
\begin{aligned}
2 \frac{1}{\sigma} e_{f}^{T} \Gamma^{-1} \dot{f} & \leq \frac{1}{\sigma} e_{f}^{T} M e_{f}(t)+\frac{1}{\sigma} \dot{f}(t)^{T} \Gamma^{-1} M^{-1} \Gamma^{-1} \dot{f}(t) \\
& \leq \frac{1}{\sigma} e_{f}(t)^{T} M e_{f}(t)+\delta,
\end{aligned}
$$

where

$$
\delta=\frac{1}{\sigma} f_{1 \max }^{2} \lambda_{\max }\left(\Gamma^{-1} M^{-1} \Gamma^{-1}\right) .
$$

If (8) holds, then

$$
\Lambda(\zeta)=\left[\begin{array}{cc}
\eta I & B^{T}(\zeta) P_{1}-F(\zeta) C \\
* & I
\end{array}\right] \pm 0 .
$$

Applying Schur complement to (16) implies that

$$
\left(F(\zeta) C-B^{T}(\zeta) P_{1}\right)\left(F(\zeta) C-B^{T}(\zeta) P_{1}\right)^{T} \prec \eta I .
$$

The minimization of $\eta$ leads to the following equality:

$$
F(\zeta) C=B^{T}(\zeta) P_{1}
$$

Hence,

$$
\begin{aligned}
\dot{V} \leq & 2 e_{x}^{T} P_{1}(A(\zeta)-L(\zeta) C) e_{x}+\frac{1}{\sigma} e_{f}(t)^{T} M e_{f}(t) \\
& +\delta-2 \frac{1}{\sigma} e_{f}^{T} B^{T}(\zeta) P_{1}\left((A(\zeta)-L(\zeta) C) e_{x}+B(\zeta) e_{f}\right) .
\end{aligned}
$$

This inequality can been rewritten as follows:

$$
\dot{V} \leq \widetilde{x}^{T} \Omega(\zeta) \tilde{x}+\delta,
$$

where $\tilde{x}=\left[\begin{array}{c}e_{x}(t) \\ e_{f}(t)\end{array}\right], \Omega(\zeta)=\left[\begin{array}{cc}\omega_{11}(\zeta) & \omega_{12}(\zeta) \\ * & \xi_{22}(\zeta)\end{array}\right]$ in which

$$
\begin{aligned}
& \omega_{11}(\zeta)=P_{1}(A(\zeta)-L(\zeta) C)+(A(\zeta)-L(\zeta) C)^{T} P_{1}, \\
& \omega_{12}(\zeta)=-\frac{1}{\sigma}(A(\zeta)-L(\zeta) C)^{T} P_{1} B(\zeta) .
\end{aligned}
$$

If (9) holds, then $\Omega(\zeta) \leq 0$. Furthermore, if (9) holds with $\varepsilon_{3}(\zeta)>0$ for $\zeta \neq 0$, then $\Omega(\zeta)<0$. Therefore, there exists a scalar $\vartheta>0$ such that

$$
\dot{V}<-\vartheta\|\widetilde{x}\|^{2}+\delta .
$$

It follows that $\dot{V}<0$ if $9\|\widetilde{x}\|^{2}>\delta$, and according to Lyapunov stability theory $\tilde{x}$ converges to the following set:

$$
S=\left\{\frac{\tilde{x}}{\|\tilde{x}\|^{2}} \leq \frac{\delta}{\vartheta}\right\} .
$$




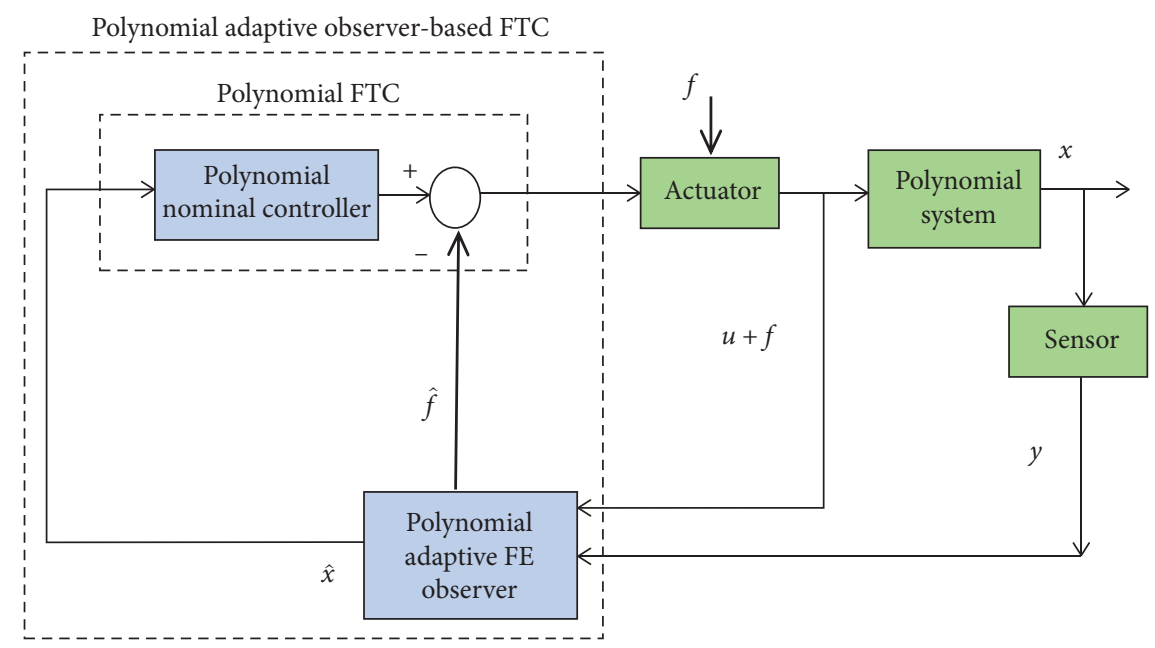

FIgUre 1: Polynomial fault tolerant control scheme.

Thus, estimation errors of both the state and the fault are uniformly ultimately bounded.

Remark 1. Selection of the learning rate $\Gamma$ influences the accuracy of the system state and actuator faults estimation (see Kharrat et al. [6]). This parameter should be adjusted such that $\delta$ is minimised.

Remark 2. The nonnegative polynomials $\varepsilon_{2}(\zeta)>0$ and $\varepsilon_{3}(\zeta)>0$ for $\zeta \neq 0$ can be accommodated by SOS optimization as in Papachristodoulou and Prajna's study. [23].

3.2. Fault Accommodation. After that, the fault information is obtained, and we will consider the fault-tolerant control design problem of system (1) to compensate the effect of actuator faults and to stabilize the resulting closed loop systems by considering the following FTC law:

$$
u=-K(\zeta) \hat{x}-\widehat{f} \text {. }
$$

Substituting (24) into (1), we obtain the following dynamic of the closed-loop system:

$$
\dot{x}=(A(\zeta)-B(\zeta) K(\zeta)) x+\rho,
$$

where

$$
\rho=B(\zeta) K(\zeta) e_{x}+B(\zeta) e_{f}
$$

$\rho$ can be considered as an external disturbance, and the boundedness of $e_{x}$ and $e_{f}$ can be guaranteed by Section 3.1. So, if the polynomial state feedback controller

$$
u=-K(\zeta) x
$$

can ensure that the following polynomial system is asymptotically stable:

$$
\dot{x}=A(\zeta) x+B(\zeta) u
$$

then state vector $x$ is uniformly ultimately bounded under observer-based fault tolerant controller (3) according to the input-to-state stability theory.

The polynomial state feedback controller (27) can be obtained by solving the SOS conditions presented in the following theorem.

Theorem 2. Control law (27) stabilizes polynomial system (28) if there exists a symmetric matrix $P_{2}$ and a polynomial matrix $W_{K}(\zeta)$ such that the following SOS conditions are satisfied:

$$
\begin{aligned}
& v_{1}^{T}\left(P_{2}-\varepsilon_{1} I\right) v_{1} \text { is SOS, } \\
- & v_{2}^{T}\left(\Upsilon(\zeta)+\varepsilon_{2}(\zeta) I\right) v_{2} \text { is SOS, }
\end{aligned}
$$

where $v_{1}$ and $v_{2}$ denote vectors that are independent of $\zeta$.

$$
\Upsilon(\zeta)=A(\zeta) P_{2}-B(\zeta) W_{K}(\zeta)+P_{2} A^{T}(\zeta)-W_{K}^{T}(\zeta) B^{T}(\zeta) .
$$

In this case, a stabilizing feedback gain $K(\zeta)$ can be obtained from $P_{2}$ and $W_{K}(\zeta)$ as $K(\zeta)=W_{K}(\zeta) P_{2}^{-1}$.

\section{Simulation Examples}

4.1. Example 1: Tunnel Diode Circuit. A tunnel diode circuit shown in Figure 2 is adopted from the study by Zhao et al. [7] and Iben Ammar et al. [16]. This electronic circuit can be described as follows: 


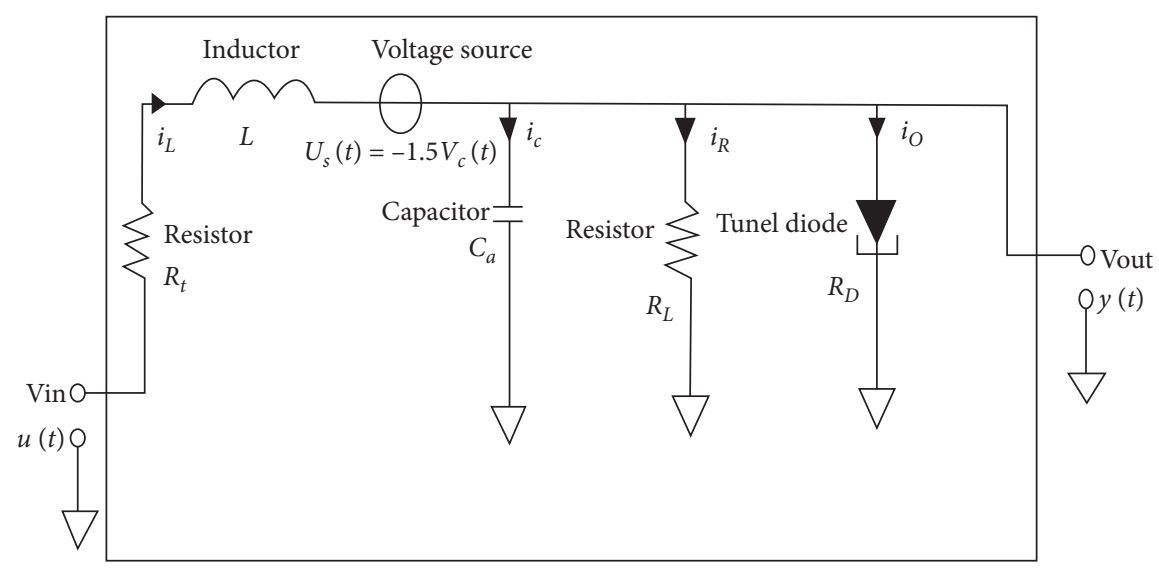

FIgURE 2: Tunnel diode circuit.

$$
\begin{aligned}
{\left[\begin{array}{c}
\dot{x}_{1} \\
\dot{x}_{2}
\end{array}\right] } & =\left[\begin{array}{cc}
\frac{1}{R_{L} C_{c}}-\frac{1}{C_{c}}\left(0.002+0.01 x_{1}^{2}\right) & \frac{1}{C_{c}} \\
\frac{1}{2 L} & -\frac{R_{E}}{L}
\end{array}\right] \times\left[\begin{array}{l}
x_{1} \\
x_{2}
\end{array}\right]+\left[\begin{array}{l}
0 \\
\frac{1}{L}
\end{array}\right] u, \\
y & =\left[\begin{array}{ll}
1 & 0
\end{array}\right]\left[\begin{array}{l}
x_{1} \\
x_{2}
\end{array}\right],
\end{aligned}
$$

where $R_{L}$ and $R_{E}$ are two resistors, $C_{c}$ is a capacitor, and $L$ is an inductor.

The tunnel diode circuit parameters are taken as

$$
\begin{aligned}
C_{c} & =25 \mathrm{mF}, \\
L & =20 \mathrm{H}, \\
R_{E} & =200 \Omega, \\
R_{L} & =2 \mathrm{k} \Omega .
\end{aligned}
$$

Based on the concept of nonlinearity sector, a T-S fuzzy model is proposed in Zhao et al. [7] to represent the dynamics of this system under $x_{1} \in\left[\bar{m}_{1} \bar{m}_{2}\right]$. In this paper, we deal directly with polynomial model (31), without any assumption. Furthermore, $x_{1}$ is not restricted to be in $\left[\begin{array}{ll}\bar{m}_{1} & \bar{m}_{2}\end{array}\right]$.

In order to illustrate the use of result, we assume that we have an actuator fault. In this case, the polynomial model is as follows:

$$
\begin{aligned}
{\left[\begin{array}{l}
\dot{x}_{1} \\
\dot{x}_{2}
\end{array}\right] } & =\left[\begin{array}{cc}
\frac{1}{R_{L} C_{c}}-\frac{1}{C_{c}}\left(0.002+0.01 x_{1}^{2}\right) & \frac{1}{C_{c}} \\
\frac{1}{2 L} & -\frac{R_{E}}{L}
\end{array}\right] \times\left[\begin{array}{l}
x_{1} \\
x_{2}
\end{array}\right]+\left[\begin{array}{l}
0 \\
\frac{1}{L}
\end{array}\right](u+f), \\
y & =\left[\begin{array}{ll}
1 & 0
\end{array}\right]\left[\begin{array}{l}
x_{1} \\
x_{2}
\end{array}\right],
\end{aligned}
$$


where $f$ is an additive actuator fault defined by

$$
f= \begin{cases}0, & 0 \leq t<5 \\ 0.5 e^{0.05(t-5)}-0.5, & 5 \leq t<80 \\ 0.5 e^{0.05 * 75}-0.5, & 80 \leq t<150\end{cases}
$$

We choose $\varepsilon_{1}=\varepsilon_{2}(\zeta)=\varepsilon_{3}(\zeta)=10^{-3}, \sigma=1$, degrees of $P_{1}, W_{L}(\zeta)$, and $F(\zeta)$ are 0,2 , and 0 , respectively. Solving the SOS conditions in Section 3.1, one can obtain that

$$
\begin{aligned}
& \gamma=0.0004, \\
& F=0.0050057, \\
& L=\left[\begin{array}{c}
1253.442 \zeta^{2}+7084.977 \\
-313.3588 \zeta^{2}-1770.6725
\end{array}\right] .
\end{aligned}
$$

The polynomial state feedback gain matrix $K(y)$ is calculated by solving SOS conditions in Section 3.2. By choosing $\varepsilon_{1}=\varepsilon_{2}(\zeta)=10^{-3}$, the degrees of $P_{2}, W_{K}(\zeta)$ are 0 and 2 , respectively. We get

$$
K=\left[2.8013 \zeta^{2}+3.6931140 .3681 \zeta^{2}+151.395\right] .
$$

By taking learning rate $\Gamma=10^{6}$ and $\sigma=1$, we obtain $\delta=25 \times 10^{-7}$. Simulation results are shown in Figures 3-5. Figure 3 shows the evolution of actuator fault and its estimated values. Figures 4 and 5 show the evolution of system states $x_{1}$ and $x_{2}$, respectively, with nominal control and fault tolerant control law.

It is noted that when actuator failures occurs, the stability of the closed-loop polynomial model with the nominal controller is not even guaranteed, whereas the closed-loop system using the fault tolerant control still operates correctly and remains maintained.

4.2. Example 2: Mass-Spring-Damper System. In this example, we consider a mass-spring-damper system (Figure 6) described by the following polynomial model given in $\mathrm{Li}$ et al. [8]:where $M$ is the mass, $x_{1}(t)$ is the displacement of the mass, $x_{2}(t)$ is the velocity of the mass, and $\mathrm{u}(\mathrm{t})$ is the input force.

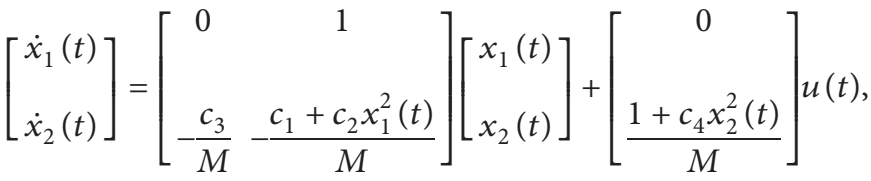

$$
\begin{aligned}
& y=\left[\begin{array}{ll}
1 & 0 \\
0 & 1
\end{array}\right]\left[\begin{array}{l}
x_{1}(t) \\
x_{2}(t)
\end{array}\right],
\end{aligned}
$$

Mass-spring-damper system parameters are $M=1$, $c_{1}=0.003, c_{2}=0.001, c_{3}=0.80$, and $c_{4}=0.1$.

We notice that the mass-spring-damper system is modeled as a polynomial system, whereas in Li et al.'s study [8], it has been modeled as an uncertain system with polytopic uncertainties by restricting the state variables $x_{1}(t)$ and $x_{2}(t)$ such as $x_{1}(t) \in\left[\begin{array}{ll}-a & a\end{array}\right]$ and $x_{2}(t) \in\left[\begin{array}{ll}-b & b\end{array}\right]$, $a>0, b>0$. However, in this paper, we do not need this restriction, we take the original model as it is.

By adding the actuator fault on the system, the polynomial model can be described by

$$
\begin{aligned}
{\left[\begin{array}{c}
\dot{x}_{1}(t) \\
\dot{x}_{2}(t)
\end{array}\right] } & =\left[\begin{array}{c}
0 \\
1 \\
-\frac{c_{3}}{M}-\frac{c_{1}+c_{2} x_{1}^{2}(t)}{M}
\end{array}\right]\left[\begin{array}{l}
x_{1}(t) \\
x_{2}(t)
\end{array}\right]+\left[\begin{array}{c}
0 \\
\frac{1+c_{4} x_{2}^{2}(t)}{M}
\end{array}\right](u(t)+f(t)), \\
y & =\left[\begin{array}{ll}
1 & 0 \\
0 & 1
\end{array}\right]\left[\begin{array}{l}
x_{1}(t) \\
x_{2}(t)
\end{array}\right],
\end{aligned}
$$




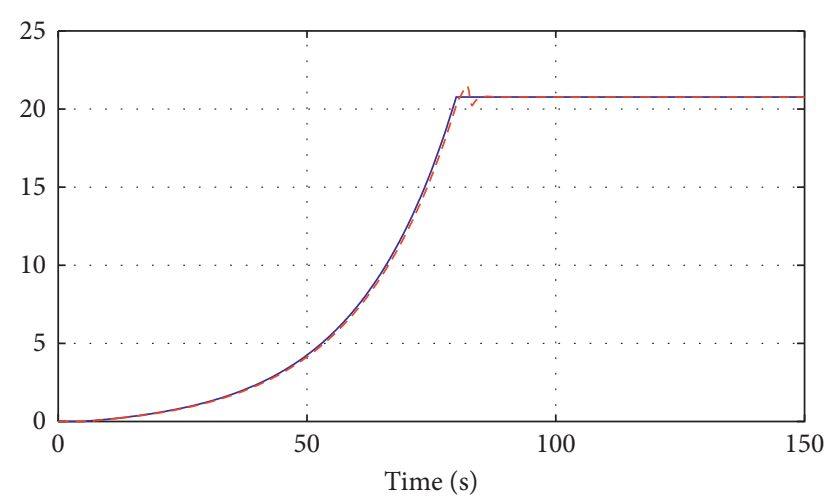

$-f$

FIgURE 3: Fault and its estimated in example 1.

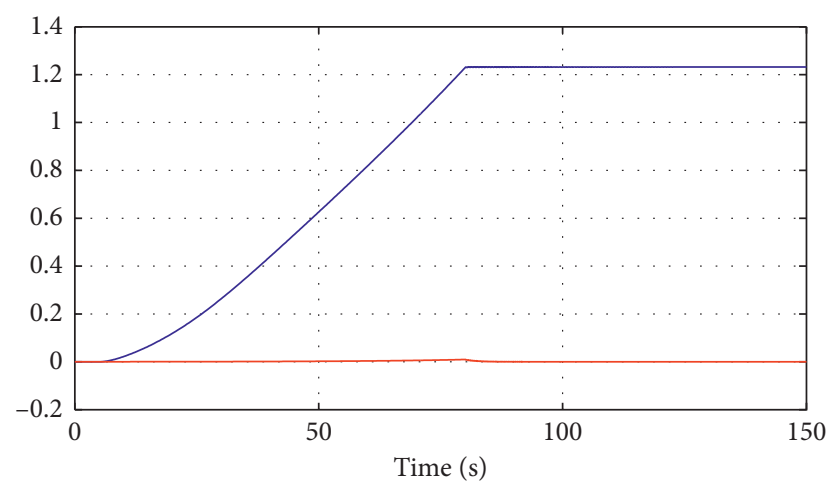

— Nominal control

— FTC control

FIGURE 4: System state $x_{1}$ with nominal control and fault tolerant control law in example 1.

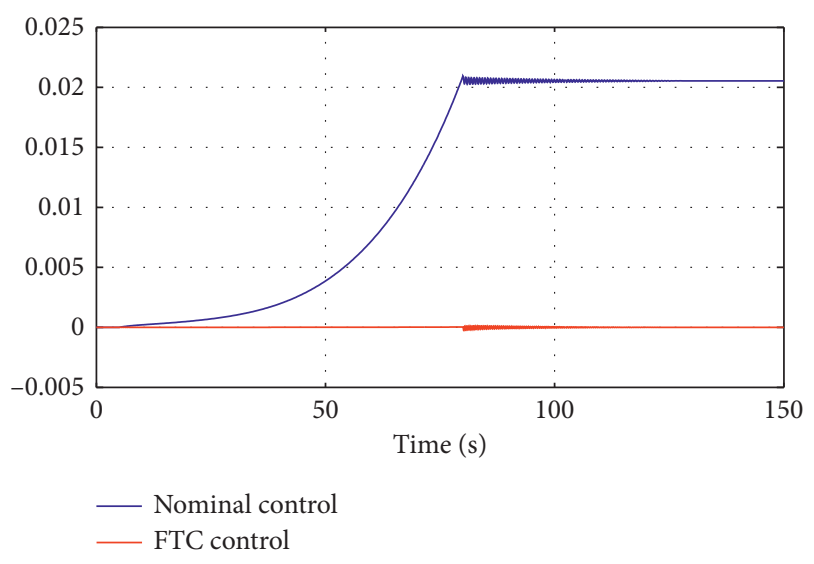

FIgURe 5: System state $x_{2}$ with nominal control and fault tolerant control law in example 1 .

where $f$ is an additive actuator fault defined by

$$
f= \begin{cases}0, & 0 \leq t<5 \\ \cos (\pi t)+2, & 5 \leq t \leq 20 .\end{cases}
$$

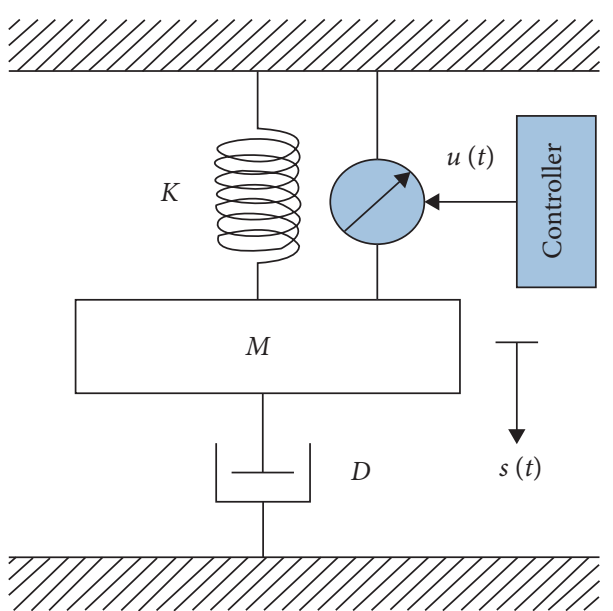

FIGURE 6: A mass-spring-damper system.

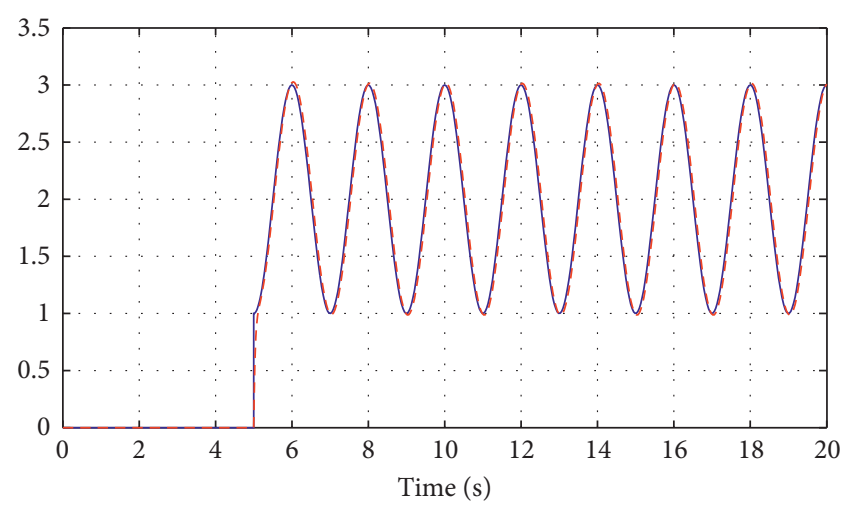

$-f$

Figure 7: Fault and its estimated in example 2.

We choose $\varepsilon_{1}=\varepsilon_{2}(\zeta)=\varepsilon_{3}(\zeta)=10^{-3}, \sigma=1, F(\zeta)$ of degree 2 in $\zeta_{2}$, and $W_{L}(\zeta)$ of degree 2 in $\zeta_{1}$. By solving SOS conditions in Section 3.1, we get

$$
\begin{aligned}
\gamma & =0.1 \times 10^{-10}, \\
F(y) & =\left[\begin{array}{lc}
0.00024962 \zeta_{2}^{2}+0.0024962 & 0.034298 \zeta_{2}^{2}+0.34298
\end{array}\right], \\
L(y) & =\left[\begin{array}{cc}
0.683066 \zeta_{1}^{2}+1.026 & 0.70759 \\
-0.0049713 \zeta_{1}^{2}-0.622816 & 0.4218721-0.001 \zeta_{1}^{2}
\end{array}\right] .
\end{aligned}
$$

Now, we choose $\varepsilon_{1}=\varepsilon_{2}(\zeta)=10^{-3}, W_{K}(\zeta)$ of degree 2 in $\zeta_{1}$. By solving the SOS conditions in Section 3.2, the polynomial controller gain is obtained as:

$$
K(\zeta)=\left[0.62 \zeta_{1}^{4}+0.52 \zeta_{1}^{2}+0.761 .3 \zeta_{1}^{4}+1.09 \zeta_{1}^{2}+1.58\right] .
$$

By choosing $\Gamma=1000$, we obtain $\delta=0.00010$. Similar to example 1, we show the evolution of actuator fault and its estimated values in Figure 7. The evolution of system state $x_{1}$ with nominal control and fault tolerant control law is given 


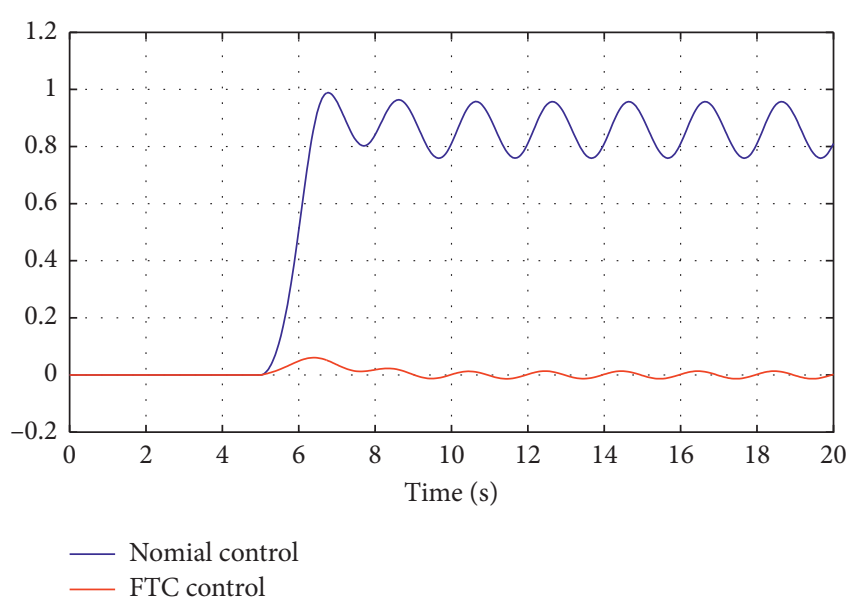

Figure 8: System state $x_{1}$ with nominal control and fault tolerant control law in example 2.

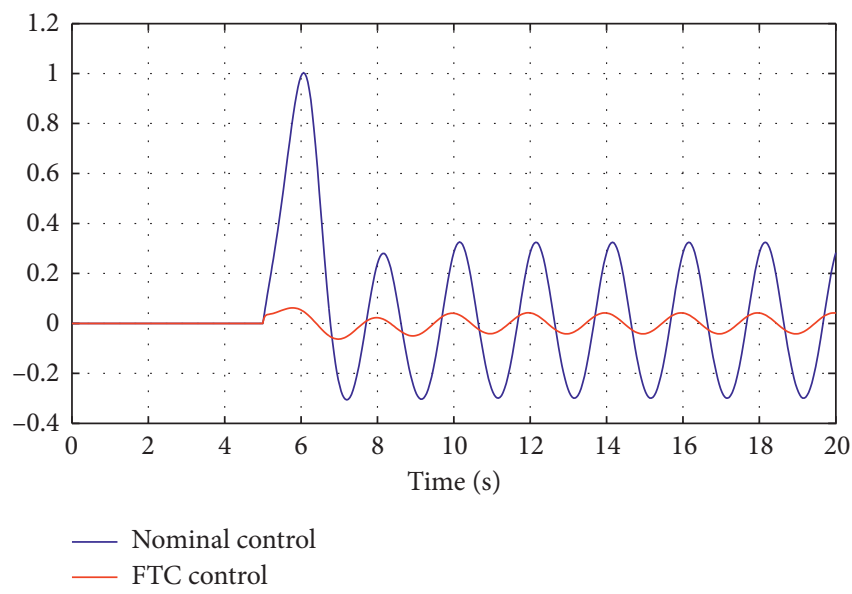

FIGURE 9: System state $x_{2}$ with nominal control and fault tolerant control law in example 2.

in Figure 8 and the evolution of system state $x_{2}$ with nominal control and fault tolerant control law in Figure 9.

\section{Conclusion}

In this paper, we have developed an adaptive actuator FTC strategy for a class of polynomial models, and sufficient analysis and design conditions in terms of SOS are proposed. Based on the adaptive fault estimation, a fault tolerant control is designed to guarantee the stability of the closed loop systems despite fault presence. Two simulation examples are presented to demonstrate the applicability of the proposed polynomial active fault-tolerant controller using the adaptive fault estimation algorithm. As future work, we will investigate the problem of adaptive fault estimation and fault-tolerant control for polynomial fuzzy systems with time delay considering measurable and unmeasurable premise variables.

\section{Data Availability}

The data used to support the findings of this study are available upon request.

\section{Conflicts of Interest}

The authors declare that there are no conflicts of interest.

\section{References}

[1] I. Elleuch, A. Khedher, and K. B. Othman, "State and faults estimation based on proportional integral sliding mode observer for uncertain takagi-sugeno fuzzy systems and its application to a turbo-reactor," International Journal of Fuzzy Systems, vol. 19, no. 6, pp. 1768-1781, 2017.

[2] Q. Jia, L. Wu, and H. Li, "Robust actuator fault reconstruction for takagi-sugeno fuzzy systems with time-varying delays via a synthesized learning and luenberger observer," International Journal of Control, Automation and Systems, vol. 19, no. 2, pp. 799-809, 2021.

[3] K. Zhang, B. Jiang, and V. Cocquempot, "Adaptive observerbased fast fault estimation," International Journal of Control, Automation, and Systems, vol. 6, no. 3, pp. 320-326, 2008.

[4] R. Tayari, A. Ben Brahim, F. Ben Hmida, and A. Sallami, "active fault tolerant control design for LPV systems with simultaneous actuator and sensor faults," Mathematical Problems in Engineering, vol. 2019, Article ID 5820394, 14 pages, 2019.

[5] D. Ichalal, B. Marx, J. Ragot, and D. Maquin, "New fault tolerant control strategies for nonlinear Takagi-Sugeno systems," International Journal of Applied Mathematics and Computer Science, vol. 22, no. 1, pp. 197-210, 2012.

[6] D. Kharrat, H. Gassara, A. El Hajjaji, and M. Chaabane, "Adaptive observer-based Ho FTC for T-S fuzzy systems. Application to cart motion model," Journal of the Franklin Institute, vol. 357, no. 17, pp. 12062-12084, 2020.

[7] D. Zhao, H. K. Lam, Y. Li, S. X. Ding, and S. Liu, "A novel approach to state and unknown input estimation for takagisugeno fuzzy models with applications to fault detection," IEEE Transactions on Circuits and Systems I: Regular Papers, vol. 67, no. 6, pp. 2053-2063, 2020.

[8] Z. Li, X. Zhao, and J. Yu, "On robust control of continuoustime systems with state-dependent uncertainties and its application to mechanical systems," ISA Transactions, vol. 60, pp. 12-20, 2016.

[9] M. Han, H. K. Lam, F. Liu, and Y. Tang, "More relaxed stability analysis and positivity analysis for positive polynomial fuzzy systems via membership functions dependent method," Fuzzy Sets and Systems, in press, 2021.

[10] Y. Zhao, H. K. Lam, G. Song, and X. Yin, "Relaxed stability conditions for polynomial-fuzzy-model-based control system with membership function information," IET Control Theory \& Applications, vol. 11, no. 10, pp. 1493-1502, 2017.

[11] C. Liu, H. K. Lam, X. Ban, and X. Zhao, "Design of polynomial fuzzy observer-controller with membership functions using unmeasurable premise variables for nonlinear systems," Information Sciences, vol. 355-356, pp. 186-207, 2016.

[12] D. Ye, NN. Diao, and X. G. Zhao, "Fault-tolerant controller design for general polynomial-fuzzy-model-based systems," IEEE Transactions on Fuzzy Systems, vol. 26, no. 2, pp. 1046-1051, 2017. 
[13] A. Chibani, M. Chadli, S. X. Ding, and N. B. Braiek, "Design of robust fuzzy fault detection filter for polynomial fuzzy systems with new finite frequency specifications," Automatica, vol. 93, pp. 42-54, 2018.

[14] H. Gassara, F. Siala, A. El Hajjaji, and M. Chaabane, "Local stabilization of polynomial fuzzy model with time delay: SOS approach," International Journal of Control, Automation and Systems, vol. 15, no. 1, pp. 385-393, 2017.

[15] H. Gassara, A. El Hajjaji, and M. Chaabane, "Control of time delay polynomial fuzzy model subject to actuator saturation," International Journal of Fuzzy Systems, vol. 18, no. 5, pp. 763-772, 2016.

[16] I. Iben Ammar, H. Gassara, A. El Hajjaji, F. Tadeo, and M. Chaabane, "Observer-based controller for positive polynomial systems with time delay," Optimal Control Applications and Methods, vol. 41, no. 1, pp. 278-291, 2020.

[17] S. Prajna, A. Papachristodoulou, and P. A. Parrilo, "Introducing SOSTOOLS: a general purpose sum of squares programming solver," in Proceedings of 41st IEEE Conference on Decision Control, pp. 741-746, Las Vegas, NV, USA, December 2002.

[18] M. Chen, H. Wang, and X. Liu, "Adaptive fuzzy practical fixed-time tracking control of nonlinear systems," IEEE Transactions on Fuzzy Systems, vol. 29, no. 3, pp. 664-673, 2019.

[19] H. Wang, W. Bai, X. Zhao, and P. X. Liu, "Finite-timeprescribed performance-based adaptive fuzzy control for strict-feedback nonlinear systems with dynamic uncertainty and actuator faults," IEEE Transactions on Cybernetics, 2021, inpress.

[20] H. Xie, z. Liu, C. Yan, and S. Zhou, "Distributed finite-time bipartite consensus for multiagent system with event-triggered control," Hindawi Mathematical Problems in Engineering, vol. 2020, Article ID 4721218, 8 pages, 2020.

[21] H. Wang, W. Bai, and P. X. Liu, "Finite-time adaptive faulttolerant control for nonlinear systems with multiple faults," IEEE/CAA Journal of Automatica Sinica, vol. 6, no. 6, pp. 1417-1427, 2019.

[22] B. Pang and Q. Zhang, "Observer-based passive control for polynomial fuzzy singular systems with time-delay via sliding mode control," Nonlinear Analysis: Hybrid Systems, vol. 37, Article ID 100909, 2020.

[23] A. Papachristodoulou and S. Prajna, "On the construction of Lyapunov functions using the sum of squares decomposition," in Proceedings of IEEE Conference on Decision and Control 2002, Las Vegas, NV, USA, December 2002. 